\title{
Utber eine Reaktion der N-Oxyde der Chinolin-Reihe mit Bleitetraacetat
}

Vor einiger Zeit haben Ochiai und Ohta gezeigt, ${ }^{1)}$ dass das Methosulfat oder das Bortrifluorid-Addukt des Chinolin-N-oxydes beim Erhitzen mit irgendeinem Metallnitrat in Dimethylsulfoxyd-Lösung das 3-Nitroderivat ergibt. Im Laufe der Fortsetzung des Versuches wurde nun bemerkt, dass Chinolin-N-oxyd (I) beim Erhitzen mit 1.2 1.5 molen Bleitetraacetat in einer Lösung von Benzol oder Chloroform ein sirupöses Produkt (II) ergibt, das beim Stehenlassen oder noch schneller beim Verseifen mit verd. Salzsäure in das kristallinische N-Hydroxycarbostyril (III) übergeht. Die Ausbeute an (III) ist befriedigend. Im Infrarot-Spektrum von (II) ist eine Bande bei $1800 \mathrm{~cm}^{-1}$ vorhanden, die der Carbonylgruppe einer N-Acetoxylgruppe zuzuschreiben ist, ${ }^{2)}$ sodass seine Konstitution als N-Acetoxycarbostyril vermutet wurde. Die Konstitution von (III) wurde durch direkten Vergleich mit dem nach Newbold und Spring ${ }^{3)}$ durch säurige Verseifung von 2Äthoxychinolin-N-oxyd hergestellten Präparat von N-Hydroxycarbostyril bestätigt.<smiles>O=[N+]([O-])c1cccc2ccccc12</smiles>

( I )

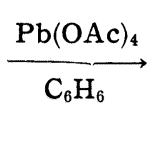

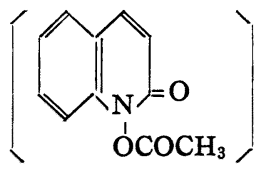

( П )

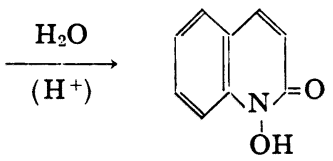

(III)

Ferner wurde gezeigt, dass die Reaktion mit Lepidin-N-oxyd, 6-Methyl-, 4-Chlorbzw. 3-Bromchinolin-N-oxyd ganz analog verläuft und das entsprechende N-Hydroxycarbostyril mit ebenso befriedigender Ausbeute ergibt, wie in Tabelle I gezeigt wird.

T ABELLE I.

Reaktionsprodukte (N-Hydroxycarbostyrile)

Chinolin-N-oxyde

Chinolin

Lepidin

6-Methylchinolin

4-Chlorchinolin

3-Bromchinolin

$\begin{array}{cll}\begin{array}{c}\text { Ausbeute } \\ (\%)\end{array} & \begin{array}{c}\text { Schmp. } \\ (\text { Form })\left({ }^{\circ} \mathrm{C}\right)\end{array} & \text { Formel } \\ 73.4 & 188 \sim 189 \text { (Prismen) }) & \mathrm{C}_{9} \mathrm{H}_{7} \mathrm{O}_{2} \mathrm{~N} \\ 81.2 & 224 \sim 226 \text { (Nadeln) } & \mathrm{C}_{10} \mathrm{H}_{9} \mathrm{O}_{2} \mathrm{~N} \\ 78.8 & 200 \sim 201 \text { (Prismen) }) & \mathrm{C}_{10} \mathrm{H}_{9} \mathrm{O}_{2} \mathrm{~N} \\ 66.4 & 200 \sim 202 \text { (Nadeln) } & \mathrm{C}_{9} \mathrm{H}_{6} \mathrm{O}_{2} \mathrm{NCl} \\ 62.6 & 187 \sim 188 \text { (Prismen) }) & \mathrm{C}_{9} \mathrm{H}_{6} \mathrm{O}_{2} \mathrm{NBr}\end{array}$

$\overbrace{\mathrm{C} \quad \mathrm{H} \quad \mathrm{N}}^{\text {Ber. }(\%)} \overbrace{\mathrm{C} \quad \mathrm{H} \quad \mathrm{N}}^{\text {Gef. }}(\%)$

$\begin{array}{llllll}68.56 & 5.18 & 8.00 & 68.51 & 5.23 & 8.23\end{array}$ $\begin{array}{lll}68.77 & 5.13 & 8.46\end{array}$

$\begin{array}{llllll}55.25 & 3.09 & 7.11 & 54.92 & 3.16 & 7.06\end{array}$

$\begin{array}{llllll}45.02 & 2.51 & 5.83 & 45.19 & 2.74 & 5.99\end{array}$

Inzwischen haben Hamana und Yamazaki gezeigt,") dass Chinolin- bzw. Lepidin-Noxyd bei der alkalischen Ferricyanid-Oxydation das entsprechende N-Hydroxycarbostyril ergeben. $\mathrm{Ob}$ der Mechanismus der oben erwähnten Reaktion mit dieser Decker'schen Oxydation analog ist, ist fraglich, denn man weiss darüber noch kaum etwas Sicheres. $\mathrm{Zu}$ bemerken ist jedoch die Tatsache, dass sich die Ausbeute an (III) auf $24 \%$ erniedrigt, wenn man die Reaktion in Eisessig-Lösung ausführt, und dass Chinaldin- $\mathrm{N}$-oxyd, das Methosulfat von (I), 4-Nitrochinolin-N-oxyd sowie Pyridin-N-oxyd der Reaktion widerstehen.

1) E. Ochiai, A. Ohta : Dieses Bulletin, 10, 349 (1962).

2) vgl. dazu C. Kaneko: Yakugaku Zasshi, 79, 428 (1959).

3) G. T. Newbold, F.S. Spring: J. Chem. Soc., 1948, 1864.

4) M. Hamana, M. Yamazaki : Dieses Bulletin, 10, 51 (1962). 
Organisch-synthetisches Laboratorium des Forschungsinstituts für physikalische und chemische Forschungen,

Kamifujimae-cho, Komagome,

Bunkio-ku, Tokio.

den 31. August 1962
Akihiro Ohta（太田明㕕）

Eiji Ochiai (落合英二) 